\title{
Diagnose Sexuelle Des Os Coxaux Archéoanthropologiques Issus De L'amas Coquillier De Songon Kassemblé Au Sud-Est De La Côte d'Ivoire
}

\author{
Coulibaly Daouda \\ Université Alassane Ouattara- Bouaké - Côted’Ivoire
}

doi: 10.19044/esj.2016.v12n17p261URL:http://dx.doi.org/10.19044/esj.2016.v12n17p261

\begin{abstract}
Since 2008, some surfaces gatherings have been done on the archaeological shells middens of Songon Kassemblé inIvory Coast. As a result, several pieces of anthropological bones have been collected. Each collection with a minimum of three individuals, contains some skulls, some longs bones of the uppers and the inferiors members, and some hips bones. These bones are the main material for experimentation. In fact, determing the sex isthe purpose of this work. To do so, firstly we will estimate the age of the hips bone owners at the time of their death. Secondly morphoscopical and morphometrical methods have been used.

The results are, the sex of three hips bone owners is female and the last one sex is indeterminate.
\end{abstract}

Keyswords: Ivory Coast, sexual diagnosis, morphoscopical and morphometrical, humans hips bones, Shell midden

\section{Résumé}

Depuis 2008, des ramassages de surface ont été effectués sur le site archéologique des amas coquilliers de Songon Kassemblé en Côte d’Ivoire. Ce qui a permis la collecte des nombreuses pièces ostéoanthropologiques. Cette collection ostéologique, dont le nombre minimum d'individus est de trois (3), comprend des crânes, des os longs des membres supérieurs et inférieurs, et des os coxaux. Ces derniers constituent le principal matériel d'étude de ce travail, dont l'objectif est la détermination du sexe des individus à qui appartiennent ces coxaux. Pour ce faire, suite à l'estimation de l'âge des personnes à qui appartiennent ces coxaux à leur décès, les méthodes morphoscopique et morphométrique ont été utilisées. Ce qui donne 
comme résultats, le sexe des propriétaires de trois coxaux est féminin, et le sexe de celui d'un coxal est indéterminé.

Mots clés : Côte d’Ivoire, diagnose sexuelle, morphoscopique et morphométrique, os coxaux humains, amas coquilliers

\section{Introduction}

La détermination du sexe est incontournable pour l'étude des vestiges ostéologiques des populations anciennes et contemporaines. Elle constitue l'un des problèmes auxquels le paléoanthropologue, le paléodémographe et le médecin légiste sont toujours confrontés. La détermination sexuelle se base sur l'existence d'un dimorphisme sexuel. Ce dernier qui s'exprime aux plans génétique, gonadique, génital et somatique, s’observe surtout au niveau des os coxaux. Notre étude ne s’intéressera qu'aux données somatiques pour déterminer le sexe des individus auxquels appartiennent les os coxaux provenant de l'amas coquillier de Songon Kassemblé.

La sexualisation individuelle somatique prend un véritable effet à partir de l'âge de la puberté sous l'action des hormones sexuelles. Ce qui amplifie donc la différence sexuelle morphologique. D’où la nécessité de procéder d'abord à une estimation de l'âge au décès des sujets à qui appartiennent notre matériel d’étude: les coxaux. Les approches morphologique et ostéométrique seront ensuite utilisées.

\section{Matériel et méthodes \\ Matériel}

\section{Du choix de l'os coxal pour déterminer le sexe}

L'échantillon de cette étude pionnière en Côte d'Ivoire, comprend 02 crânes ; 18 fragments humérus dont 7 droits, 8 gauches et 3 indéterminés ; un radius droit et un gauche ; 5 fragments d'ulna dont 2 droits et 3 gauches ; 33 fragments de fémurs dont 9 droits, 12 gauches et 12 indéterminés; 14 fragments de tibias dont 1 droit, 7 gauches et 6 indéterminés; et 04 os coxaux de squelettes humains (Fig. I). Ces os sont tous issus du site des amas coquilliers en danger de Songon Kassemblé (Coulibaly et al 2011A et B ; Coulibaly 2012 et 2015).

De toutes ces pièces osseuses, seuls les os coxaux permettent de déterminer le sexe des adultes avec une probabilité supérieure à 95\% (Bruzek 2002 ; Murail et al 2005). Le sexe, donnée biologique essentielle, permet d'aborder pour les populations du passé les questions relatives au recrutement, à la morphologie, aux activités différentes entre les sexes, aux pratiques funéraires. Les hommes et les femmes n'étant pas accompagnés du même type de mobilier, de parure. L'architecture des tombes peut-être parfois différente. 
Les autres critères osseux "extra-coxaux" sont liés à la robustesse et ils ont une relation beaucoup moins étroite avec le sexe de l'individu. Les os tels que les crânes, et les os longs sont beaucoup moins performants. En outre, leur utilisation pour faire une diagnose sexuelle, nécessite une certaine expérience, que possède l'anthropologue confirmé. Autant de raisons qui motivent en conséquence notre choix pour les os coxaux.

\section{Présentation des os coxaux}

L’os coxal gauche (fig.1-A) en vue médiale est représenté par toutes ses parties, à l'exception de la branche inférieure du pubis et de la branche de l'ischium. Ces deux branches sont fragmentées.L'os coxal droit (fig.1-B), en vue médiale, a un très bon état de conservation. Il ne présente aucune fragmentation. En revanche, les deux autres coxaux de notre matériel d'étude sont beaucoup fragmentés. La figure 1-C est un fragment de coxal gauche toujours en vue médiale. Il comprend une partie de l'ilium et de l'acétabulum, précisément sa fosse acétabulaire. Quant à la figure 1-D, le fragment de coxal droit en vue médiale, qu'elle présenteest composé : d'une part, du pubis représenté par le corps, de la branche supérieure et de la branche inférieure en partie ; et d'autre part, d'une partie dela surface semilunaire acétabulaire. Les mensurations de ces os coxaux sont réalisées avec un pied à coulisse (Deto, L.200mm, précision 0.02mm) et un ruban métrique.

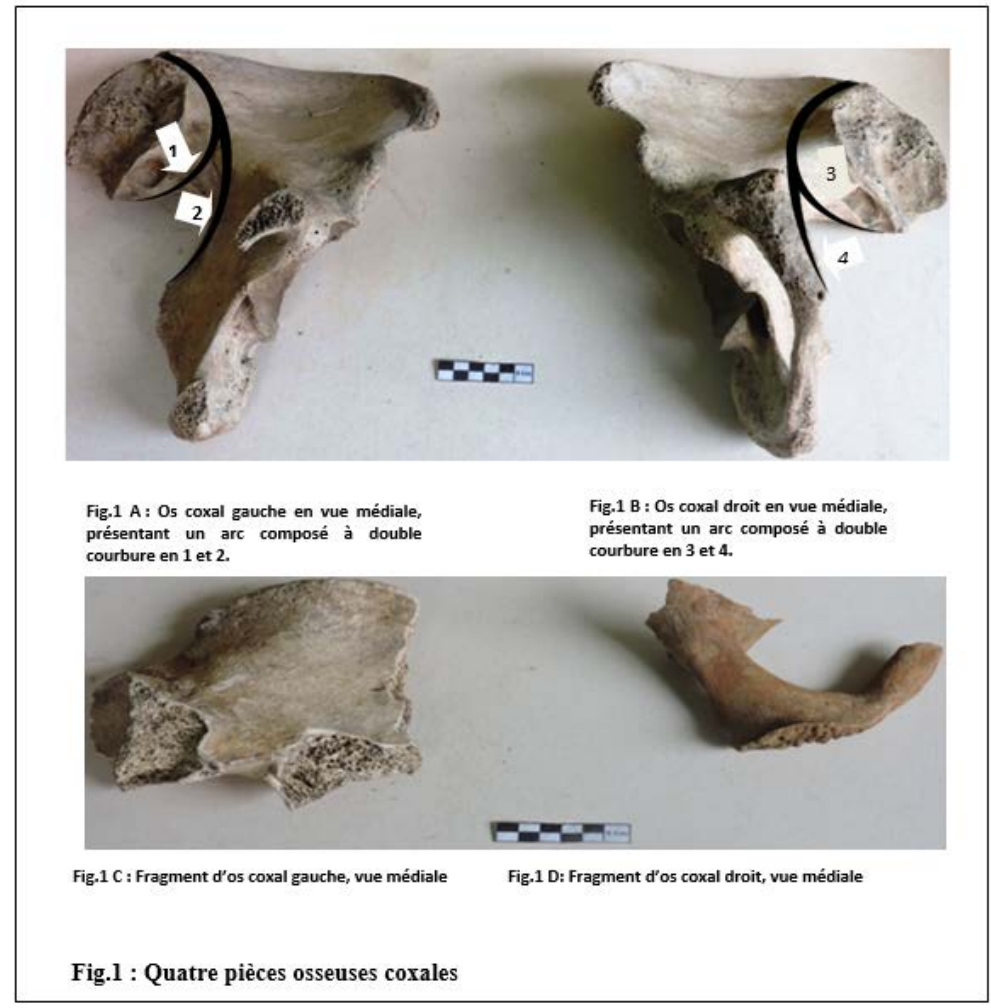


as: Surface auriculaire

sn: Grande incisure ischiatique

$\mathrm{AB}$ : Largeur de la grande incicure ischiatique

CD: Profondeur de la grande incisure ischiatique

$\mathrm{AC}$ : Segment supérieur de la largeur $\mathrm{AB}$

$\mathrm{AP}$ : Parallèle à la profondeur CD

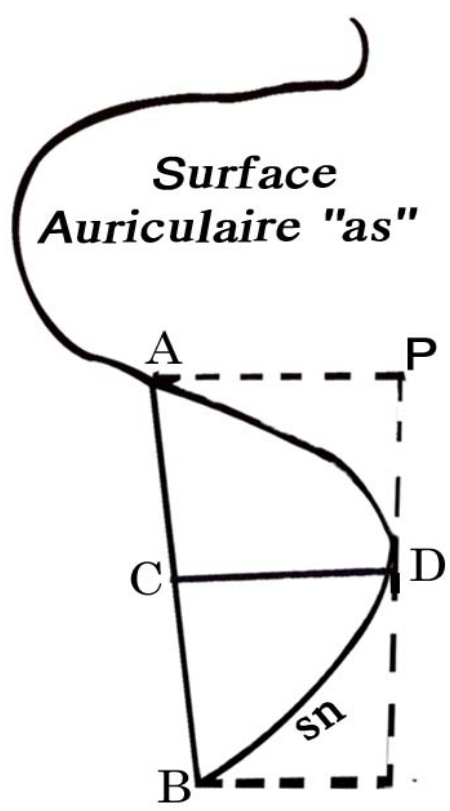

Fig.2A: Symétrie par rapport à la ligne de plus grande profondeur de la grande incisure ischiatique du coxal droit (Fig.1A).

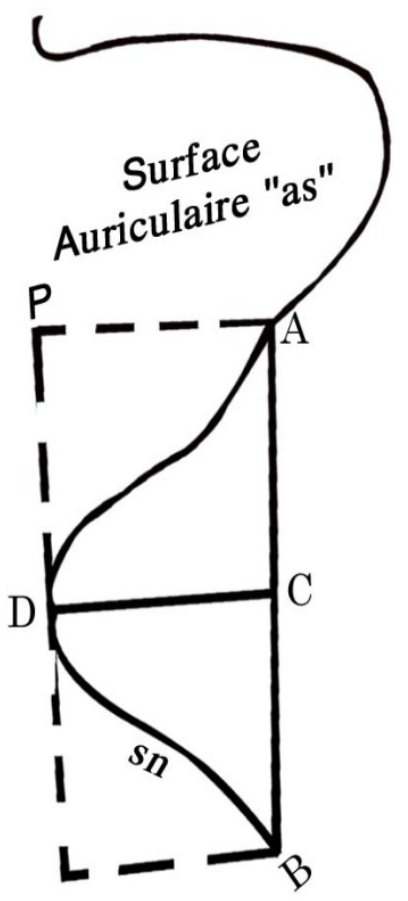

Fig.2B: Symétrie par rapport à la ligne de plus grande profondeur de la grande incisure ischiatique du coxal gauche (Fig.1B). 


\section{De la méthode d'observation morphologique à la méthode d'observation morphométrique}

Deux méthodes ont été utilisées afin d'aboutir à une diagnose sexuelle fiable : l'observation morphologique ${ }^{1}$ des caractères sexuels de l'os coxal (Bruzek 2002) et l'approche morphométrique ${ }^{2}$ de l’os coxal (Murail et al 2005).

\section{Méthode d'observation morphologique}

La méthode morphoscopique admet plusieurs limites (Bruzek et al 2005; Murail et al 2005) :

- elle est difficile à appliquer car nécessitant des coxaux entiers. En outre, avec elle la diagnose sexuelle est impossible au cas où les traits féminins et masculins considérés sont également représentés et aussi en présence d'individus, dont la sexualisation somatique est trop modérée.

Cette méthode ne s’applique, en général, qu’aux coxaux entiers des sujets ayant atteint leur maturité biologique, à savoir après 18-20 ans. Toutefois, le sujet peut-être plus jeune. Dans ce cas, il faut que les 3 parties de l'os coxal soient soudées. Par conséquent, une estimation de l’âge des coxaux $^{3}$ fig.1-A et fig.1-B est donc incontournable. Surtout que cet os possède des caractères qui permettent d'estimer l'âge au décès des adultes. Les enfants sont exclus de ce schéma (Majo 2000).

\section{Estimation de l’âge des coxaux fig.1 A et fig.1-B}

Cette estimation de l'âge se fait à partir de leurs points d'ossification primaires et secondaires. Trois points d'ossification primaires pour les trois parties principales : l'ilium, l'ischium et le pubis, se distinguent. L'ischium et le pubis fusionnent d'abord autour de 10 ans. La fusion à l'ilium intervient ensuite au début de l'adolescence, à partir de 15 ans $^{4}$ (Bruzek et al 2005). La synostose de ces trois os primitifs se fait à l'intérieur de l'acétabulum vers 15-16 ans (Platzer 2007).

Ces deux coxaux présentent aussi tous leurs points d'ossification secondaires: la crête iliaque, la tubérosité de l’ischium, l’épine iliaque antéro-inférieure, l'épine ischiatique, le tubercule pubien et les points de l'acétabulum. Ce qui montre que la maturation osseuse des sujets à qui

${ }^{1}$ Observation morphologique ou approche morphoscopique.

2 Approche morphométrique utilise les mesures coxales de la «diagnose sexuelle probabiliste-DSP ».

${ }^{3}$ Ces coxaux présentent leurs principales parties : l'iluim, le pubis et l'ischium. Leur état de conservation est donc bon. En revanche, la méthode morphologique ne peut s'appliquer aux coxaux fig.1-C et fig.1-D car ils sont très fragmentés. L'estimation de leur âge n'est donc pas nécessaire.

4 Selon Bruzek et al 2005, p.236, par convention, l'âge des adolescents en paléoanthropologie, est compris entre 15 et 20 ans. 
appartiennent ces coxaux est achevée. Par exemple, la synostose respective de la tubérosité de l'ischium et de la crête iliaque qui apparaissent à la puberté, se fait vers 20 ans, et entre 20 et 30 ans (Murail 2010 ; Crubézy et al 2005). Nous sommes donc en présence de coxaux de sujets adultes. Au regard des insuffisances de la méthode morphoscopique, la méthode morphométrique a été employée.

\section{Méthode morphométrique}

Cette méthode présente les avantages suivants:

- confirmer ou infirmer la méthode morphologique surtout que nous nous initions à l'observation visuelle des coxaux pour effectuer une diagnose sexuelle ;

- diminuer davantage le pourcentage de sujets non sexés ;

- améliorer la fiabilité des résultats ;

- disposer de plus d'approches possibles afin de faire face au caractère très fragmentaire de certains coxaux issus d'un contexte archéologique (Marchal 2003), comme les coxaux des fig.1-C et fig.1-D.

L'os coxal n'a donc pas besoin d'être complet. En l'absence du pubis, bien qu'il porte de majeurs caractères osseux, la détermination du sexe dans ce contexte peut se faire.

\section{Spécificité de ces deux méthodes}

Ces deux méthodes ont enfin, la particularité de s’appliquer à l'ensemble des segments de l'os coxal. On constate que le dimorphisme exprimé par la totalité de l'os coxal est représentatif du dimorphisme adaptatif, commun au sein de l'espèce humaine. En conséquence, englobant un dimorphisme global des coxaux, ces méthodes ne peuvent être spécifiques à une population donnée. La fiabilité de leur application à l'échelle de tout Homo sapiens sapiens est ainsi assurée. On note cependant que parfois l'approche morphoscopique ne permet pas une détermination fiable, dans moins de $10 \%$ des cas, comme dans l'approche morphométrique avec la DSP (Bruzek et al 2005 ; Murail et al 2005). Aucune de ces deux méthodes n’a vocation à primer sur l'autre. La détermination du sexe des propriétaires des quatre coxaux de notre collection ostéologique peut, ceci dit, à présent se faire. 


\section{Résultats de la détermination du sexe Approche morphoscopique}

Cette méthode est traduite par une observation de cinq caractères présents sur l'iluim, l'ischium et le pubis. Ce sont: la région préauriculaire, la grande incisure ischiatique, l'arc composé, le bord inférieur et la longueur relative du pubis et de l'ischium. L'observation morphologique de ces cinq caractères répartis sur les trois segments des coxaux gauche (fig.1-A) et droit (fig.1-B) donne les résultats suivants :

- la région préauriculaire présente une dépression nette caractérisée par une circonférence fermée. En revanche, cette région ne présente pas de tubercule buisson. Ce qui traduit la trace d'un traumatisme obstétrical de fréquence variable. La grande incisure ischiatique est caractérisée par une symétrie par rapport à la ligne de plus grande profondeur. Cette symétrie (fig.2A et fig.2B, p.264) s'observe au niveau de la grande incisure ischiatique des coxaux : gauche (fig.1-A) et droit (fig.1-B).

- L’arc composé présente une double courbe, dont une au niveau de l'incisure et l'autre au niveau de la surface auriculaire. Sur le bord inférieur, la branche inférieure du coxal droit (fig.1-B) présente une éversion externe et est dépourvue de crista phallica. Cette observation ne peut se faire sur le coxal gauche (fig.1 A) car sa branche inférieure est fragmentée.

- Enfin, en ce qui concerne la longueur relative du pubis et de l'ischium : les pubis des deux coxaux sont plus longs que leurs ischiums. En effet, le pubis du coxal gauche (fig.1 A) mesure environ $6.6 \mathrm{~cm}$, quand son ischium fait environ $6.1 \mathrm{~cm}$. Le coxal droit (fig.1-B) a un pubis long de 7.1 $\mathrm{cm}$ et un ischium mesurant $5.8 \mathrm{~cm}$.

Au regard de ce qui précède, nous sommes en présence d'os coxaux de sexe féminin pour le coxal droit (fig.1-B) et pour le coxal gauche (fig.1 A). Les coxaux fig.1-C et fig.1-D sont à majorité fragmentés. Ce qui rend impossible l'observation des cinq caractères osseux précités. La méthode d'observation morphologique ne peut donc se faire que sur les coxaux (fig.1A) et (fig.1-B). En effet, ils sont dans un bon état de conservation. Cette diagnose sexuelle est-elle vérifiée avec la méthode morphométrique ?

\section{Approche morphométrique : DSP}

Les résultats obtenus en utilisant le logiciel de DSP, conçu par les chercheurs : Murail (P), Bruzek (J), Houët (F) et Cunha (E) (2005) sont les suivants : 


\begin{tabular}{|c|c|c|c|c|c|c|c|c|c|c|c|c|c|}
\hline Coxal & Pum $^{56}$ & Spu & Dcox & IImt & Ismm & Scox & Ss & Sa & Sis & Veac & PF & PM & SEX \\
\hline $\begin{array}{c}\text { Gauche } \\
\text { fig.1- } \\
\text { A) }\end{array}$ & ND & 30.50 & ND & 56.00 & 100.00 & 152.00 & 75.50 & 81.5 & 37.50 & 58.00 & 0.9823 & 0.017 & Female \\
\hline $\begin{array}{c}\text { Droit } \\
\text { fig.1- } \\
\text { B) }\end{array}$ & 66.00 & 31.50 & 200.00 & 60.00 & 102.00 & 151.00 & 73.50 & 82.5 & 39.00 & 58.00 & 0.9984 & 0.0016 & Female \\
\hline $\begin{array}{c}\text { Gauche } \\
\text { fig.1- } \\
\text { C) }\end{array}$ & & & & & 12.40 & 79.00 & 77.00 & & 42.00 & 1,0000 & 0,0000 & Female \\
\hline $\begin{array}{c}\text { Droit } \\
\text { (Fig.1- } \\
\text { D) }\end{array}$ & 57.00 & & & & & & & & & & & ND \\
\hline
\end{tabular}

Comment peut-on analyser ces résultats ?

\section{Analyse}

\section{Fragmentation importante des coxaux : frein à la diagnose sexuelle}

Le coxal gauche (fig.1-A): Pum et Dcox sont indéterminées car le pubis du coxal gauche est dépourvu des $3 / 4$ de ses parties. On note l'absence de la branche inférieure, de la crête pubienne, du tubercule et du pecten pubiens, et de la surface symphysaire. Seule la branche supérieure du pubis est en partie présente. Ce qui n’a pu empêcher la détermination de son sexe.

La fragmentation très importante du coxal gauche (fig.1-C) et du coxal droit (fig.1-D) n'ont pas permis de leur appliquer, comme il se doit, les méthodes ci-dessus citées. En effet: le coxal (fig.1-C) est représenté seulement que par une partie de l'ilium, comprenant l'acétabulum en particulier la fosse acétabulaire. Cette partie est dépourvue de la facette auriculaire, de la tubérosité iliaque, de la crête iliaque et de l'épine iliaque antérieure et supérieure. Néanmoins, bien qu'ayant subi une fragmentation

${ }^{5} 1^{5}$ - Pum (M.14) : longueur pré-acétabulaire du pubis: comprise entre le symphysion et le point le plus proche du bord de l'acétabulum (Bräuer 1988).

2- Spu (M.14.1) : Largeur pubo-acétabulaire (Bräuer 1988).

3- Dcox (M.1) : Hauteur de l'os coxal (Bräuer 1988).

4- Iimt (M.15.1) : Hauteur de la grande incisure ischiatique (Bräuer 1988).

5- Ismm : Longueur de l'ischium post-acétabulaire maximale (Schulter-Ellis et al. 1983).

6- Scox (M. 12) : Largeur de l'ilium (Bräuer 1988).

7- Ss : Distance spino-sciatique (Gaillard 1960).

8- Sa : Distance spino-auriculaire (Gaillard 1960).

9- Sis : Largeur cotylo-sciatique (Murail et al 2005).

10- Veac (M.22) : diamètre vertical de l'acétabulum (Bräuer 1988).

11- PF : Probabilité d'être féminin (Murail et al 2005).

12- PM : Probabilité d'être masculin (Murail et al 2005).

13- SEX : Sexe.

14- ND : Indéterminé

${ }^{6} 1$ à 14 confère Murail et al 2005, p.170. 
très importante de ses principales parties, la méthode morphométrique a permis sa diagnose sexuelle. Il s'agit d'un coxal féminin.

Quant à l’os coxal droit (fig.1-D), il n’est représenté que par la branche ischiatique qui forme avec la branche inférieure du pubis, la limite inférieure du foramen obturé. La tubérosité ischiatique présente conduit jusqu'à une partie de la surface semi-lunaire de l'acétabulum. En conséquence, seule la longueur pré-acétabulaire du pubis (Pum), comprise entre le symphysion et le point le plus proche du bord de l'acétabulum, a pu être relevée.

Ce qui ne permet pas la diagnose sexuelle de ce coxal avec chacune des deux méthodes utilisées. L'anthropobiologie virtuelle et l'anthropobiologie moléculaire sont donc les bienvenues pour reconstituer non seulement ces coxaux fragmentés, mais aussi pour déterminer le sexe du coxal que les méthodes morphoscopique et morphométrique ne permettent pas de faire.

\section{L'anthropobiologie virtuelle et l'anthropobiologie moléculaire : alternatives aux limites des méthodes morphologique et morphométrique}

L'anthropobiologie virtuelle : de l'imagerie anatomique aux 3D

L'anthropobiologie virtuelle présente plusieurs avantages pour notre collection osseuse. Ce sont: la visualisation des structures anatomiques internes, l'obtention de mesures fixes, la reconstitution par symétrie des parties anatomiques manquantes associée à la combinaison virtuelle des fragments, la correction des déformations, les analyses morphologiques bidimensionnelles ou tridimensionnelles ${ }^{7}$, l'impression en 3D des coxaux, et l'obtention de données numériques. Ainsi, la métrique de toutes les parties anatomiques fragmentées sur les coxaux : gauches (fig.1-A et 1-C) et droit (fig.1-D) pourra se faire.

\section{L’anthropobiologie moléculaire}

La méthode de la biologie moléculaire est une des perspectives pour déterminer le sexe du coxal droit (fig.1-D) de la collection osseuse étudiée ici. En effet, ce coxal est en majeure partie fragmenté. L’anthropobiologie moléculaire par le biais de la technique de la Polymerase chain Reaction (PCR) permettra par exemple, l'amplification des séquences des chromosomes sexuels $\mathrm{X}$ et $\mathrm{Y}$ afin de déterminer le sexe du coxal droit (fig.1D). Cette méthode a pour avantage de confirmer ou infirmer les résultats de celle de l'anthropobiologie virtuelle.

7 Elle permet donc l'obtention des images virtuelles bidimensionnelles (2D) ou tridimensionnelles (3D). 


\section{Conclusion}

Les méthodes d’observation morphologique et ostéométrique permettent de déterminer sans difficulté le sexe des coxaux bien conservés. Afin d’éviter les biais importants des résultats, et qui vont impacter négativement le reste de l’étude, la méthode morphométrique se positionne comme une précieuse alternative à la méthode morphoscopique. En revanche, bien qu'onéreuses les méthodes d'anthropobiologie virtuelle et d'anthropobiologie moléculaire sont de nature à combler les insuffisances des méthodes morphologiques et morphométriques, et d'infirmer ou de confirmer leurs résultats.

\section{Références:}

BRUZEK (J) 2002, " A method for visual determination of sex, using the human hip bone», in American Journal of Physical Anthropology, n¹17, p.157-168.

BRUZEK (J), SCHMITT (A) et MURAIL (P) 2005, «Identification biologique individuelle en paléoanthropologie. Détermination du sexe et estimation de l’âge au décès à partir du squelette », in Objets et Méthodes en Paléoanthropologie, Paris, CTHS, p.217-246.

COQUEUGNIOT (H) et DUTAILLY (B) 2010, "Anthropologie - imagerie médicale et 3D », in De Néandertal à l'Homme Moderne. L'Aquitaine préhistorique, vingt ans de découvertes (1990-2010), Bordeaux, Editions confluences, p.66-67.

COQUEUGNIOT (H), DESBARATS (P) et DUTAILLY (B) 2010, « Anthropologie - du virtuel au réel : l’impression 3D », in De Néandertal à l'Homme Moderne. L'Aquitaine préhistorique, vingt ans de découvertes (1990-2010), Bordeaux, Editions confluences, p.68-69.

COULIBALY (D) 2015, «Anthropobiologie des collections ostéoanthropologiques de l'amas coquillier de Songon kassemblé : premières approches ", in Les cahiers de l'Irda n 003, Premier semestre, p.231-251.

COULIBALY (D) 2012, Les collections ostéoarchéologiques humaines en Côte d'Ivoire: de leur conservation à l'analyse archéothanatologique et anthropobiologique. Thèse unique de doctorat en anthropologie, Université Félix Houphouët-Boigny, Abidjan-Coccody, Côte d’Ivoire, 349 p.

COULIBALY (D), BEUGRE (J.B), KAKOU (S), DJAHA (K), COURTAUD (P) 2011A, «Les vestiges archéo-ostéologiques humains en Côte d'Ivoire : un patrimoine anthropologique à conserver. ", in Kasa Bya Kasa. Rev.ivoir.anthropol.sociol n 19, Abidjan, EDUCI, p.33-43.

COULIBALY (D), KOUASSI (K.S), COURTAUD (P), KIENON(K.T.H) 2011B, « Méthode d'approche pour une collecte d'ossements archéoanthropologique dans l'amas coquillier en danger de Songon Kassemblé : un cas de collaboration entre archéologue et paléanthropologue en Côte 
d'Ivoire. ", in Nyansa-pô, revue Africaine d'Anthropologie n¹1, Abidjan, EDUCI, p.22-33.

DEBONO (L), MAFART (B), GUIPERT(G), et JEUSEL (E), « Application pratique de la méthode d'estimation de l’âge au décès de Schmitt et Broqua (2000) » 2004, in Bulletins et Mémoires de la Société d'Anthropologie de Paris [En ligne], T.16, Fascicule 1-2. http://bmsap.revues.org/document599.html. Mis en ligne le: 13 mai 2008. FEREMBACH (D), SCHWIDETZKY (I) et STLOUKAL (M) 1979, « Recommandations pour déterminer l'âge et le sexe sur le squelette ", in Bulletins et Mémoires de la Société d'Anthropologie de Paris, T.6, Série XIII, p.7-45.

MAJO (T) 2000, L'os coxal non adulte: Approche méthodologique de la croissance et de la diagnose sexuelle. Applications aux enfants du Paléolithique moyen. Thèse en sciences Biologiques et Médicales. Université Bordeaux I.

MARCHAL (F) 2003, «Le dimorphisme sexuel de conformation de l'os coxal humain. Bases biologiques et nouvelles applications possibles », in Bulletins et Mémoires de la Société d'Anthropologie de Paris [En ligne], T.15, Fascicule 1-2. http://bmsap.revues.org/document552.html. Mis en ligne le : 09 janvier 2006.

MURAIL (P) 2010, Ostéologie humaine et paléobiologie. Ostéologie infracrânienne. Cours du Master d’Anthropologie Biologique et Préhistoire, Bordeaux, France, 58 p.

MURAIL (P), BRUZEK (J), HOUËT (F) et CUNHA (E) 2005, « DSP : A tool for probabilistic sex diagnosis using worldwide variability in hip-bone measurements ", in Bulletins et Mémoires de la Société d'Anthropologie de Paris, n.s., T.17, 3-4. p.167-176.

PLATZER (W) 2007, Atlas de poche d'anatomie. Appareil locomoteur (4è édition), Paris, Flammarion Médecine-Sciences, 461p.

SCHMITT (A) 2005, «Une nouvelle méthode pour estimer l'âge au décès des adultes à partir de la surface sacro-pelvienne iliaque », in Bulletins et Mémoires de la Société d'Anthropologie de Paris [En ligne], T.17, Fascicule 1-2.http://bmsap.revues.org/document943.html. Mis en ligne le : 03 janvier 2008.

SUSANNE (C), POLET (C) (S/d) 2005, Dictionnaire d'anthropobiologie, Bruxelles, Editions De Boeck Université, 401p.

VILLENA I MOTA (N), DUDAY (H), HOUËT (F) 1996, « De la fiabilité des liaisons ostéologiques ",in Bulletins et Mémoires de la Société d'Anthropologie de Paris, t 8, (3-4), p.373-384.

VILLENA I MOTA (N) 1997, «Les liaisons ostéologiques et leurs applications», in Bulletins de la Société d'Anthropologie du Sud-Ouest, p.111. 